Chronic Obstructive Pulmonary Diseases:

Journal of the COPD Foundation

COPD

FOUNDATION

\title{
Perspective
}

\section{Websites, Online Communities and Digital Channels as a Medium to Effectively Educate, Engage and Empower Patients}

\author{
Elisha Malanga, $\mathrm{BS}^{1}$ Vincent Malanga, BS, $\mathrm{MCP}^{1}$ Sara Latham, $\mathrm{BA}^{1}$
}

\begin{abstract}
Abbreviations: chronic obstructive pulmonary disease, COPD; nontuberculous mycobacterial, NTM Date of Acceptance: October 16, 2018

Citation: Malanga E, Malanga V, Latham S. Websites, online communities and digital channels as a medium to effectively educate, engage and empower patients. Chronic Obstr Pulm Dis. 2018;5(4):334-337. doi: https://doi.org/10.15326/jcopdf.5.4.2018.0158
\end{abstract}

\section{COPD Foundation, Washington, DC}

\section{Address correspondence to:}

Elisha Malanga

COPD Foundation

Email: emalanga@copdfoundation.org

Phone: 866-731-2673 x309

\section{Keywords:}

online communities; websites; digital channels; patient education; patient engagement

\section{Introduction}

Most of us will agree that online access has changed the way most individuals conduct their day-to-day lives compared to only 10 years ago, but has access to online health care information revolutionized the way individuals approach their health and health care? Immediate access to information has birthed a generation of educated, engaged and empowered patients like never before. ${ }^{1}$ Results in a 2013 Pew Research Center report indicate that of the $85 \%$ of adults in the United States who use the Internet, $72 \%$ (or about $61 \%$ of all U.S. adults) reported using it to find information about their health. ${ }^{2}$ In addition, the evolution of the online community with peer-topeer support and education has provided a wealth of resources as well as complexity to the landscape. Individuals participating in online communities report significant benefit from using the communities and the usage continues to increase. ${ }^{1}$ The question that comes to mind is no longer whether the increased presence of online health education will be utilized, but rather: Is there evidence to suggest that we should not just "accept the existence of" online health care communities-but rather, embrace them for the education, peer-to-peer support and other opportunities they offer?

A recent article published in the Journal of the COPD Foundation was designed to collect data on where patients are seeking information on their health and how well online sources addressed their specific needs. ${ }^{3}$ According to the article, 445 individuals with chronic obstructive pulmonary disease (COPD) who responded to an online survey reported that physicians were their primary source of information about their disease with internet searches following as their second most frequent source of information. ${ }^{3}$ This group further reported that their online searches for COPD information focused on 2 areas: "specific information about COPD" and "treatments for the disease."3

In January 2017, IntakeQ reported that the Pew Research Center found that 1 in 4 Internet users who also reported living with a chronic disease (or having a loved one with a chronic disease) have sought others with similar health concerns on the web. ${ }^{4}$ In addition, the Pew Research Centers online article The Online Healthcare Revolution, reports that 52 million Americans have sought information online about their health and of these "health seekers," as described in the article, 21 million of them indicated that what they read online swayed their decision making. ${ }^{5}$ With the substantial impact that online learning and communities are having on patients, physicians recognize the value of the online community, especially to those with chronic diseases like COPD, but still 
remain concerned about the source and validity of the information. ${ }^{6}$ In addition, data privacy and ownership of online data has become a top concern for all online users.

The question of validity of sources has led to other questions not only about the source itself, but whether websites are updated regularly and how online communities are monitored and moderated. Various entities including industry, for-profit companies and advocacy organizations including the COPD Foundation, have developed online tools and interactive websites to address the increasing desire for quality online engagement. The breadth of information can be overwhelming and begs the question how does a patient with a chronic illness like COPD choose the right online information source and community?

The COPD Foundation struggled with this question and in 2014, after extensive research and input from our community of patients, caregivers, physicians and internal staff, launched COPD360social, an online community and home for individuals with COPD, their families, caregivers and health care providers. In 2016, in response to an unmet need in the bronchiectasis and nontuberculous mycobacterial (NTM) community, the COPD Foundation launched BronchandNTM360social, which includes the same framework, features and capabilities detailed in the context of COPD360social below.

To facilitate the goal of making COPD360social usable and efficient, the COPD Foundation chose to integrate COPD360social directly into the COPD Foundation website, providing users seamless access to all COPD Foundation resources and interactive community features. The website has features to search content by topics and dynamically publish information useful to the community on a regular basis. This functionality aims to provide an engaging, interactive and complete user experience. Current membership includes more than 35,000 members from 139 countries with more than $65 \%$ of the community comprised of individuals personally impacted by COPD by virtue of being a patient, caregiver, friend or family member (Table 1). Interestingly, prior to the launch of the online community, $80 \%$ of all registered members to the Foundation website were health care providers registering for access to educational materials. With the inception of the online community, patients and those within their circle now had a reason to register to gain access not merely to content but

\section{Table 1. COPD360social Registration Statistics $^{\mathrm{a}}$}

\begin{tabular}{rrll}
\multicolumn{1}{c}{$\begin{array}{l}\text { Registered Percentage } \\
\text { Users }\end{array}$} & \multicolumn{2}{|}{ Uf Users } & \\
\hline 2016 & $6.48 \%$ & Caregiver \\
\hline 9930 & $31.93 \%$ & Medical Affiliate/ Healthcare Professional \\
\hline 1150 & $3.70 \%$ & Friend \\
\hline 15021 & $48.30 \%$ & Patient \\
\hline 2980 & $9.58 \%$ & Family Member \\
\hline
\end{tabular}

${ }^{\mathrm{a}}$ As of September 10, 2018. Users represented from 139 countries

to a supportive and continually evolving community of peers. This engagement has been reflected in the volume of questions posed: to date, nearly 2000 questions have been asked by individuals, including more than 1000 in the past year.

COPD360social is managed by the COPD Foundation, not a third-party vendor, like many other online communities. What does this mean to its users? This was designed to provide assurance to the users of the platform that the COPD Foundation will, in accordance with its mission, protect the interest of the patient and provide reputable, unbiased content. All educational materials and related references are developed by COPD Foundation staff in conjunction with oversight and input from either its Medical and Scientific Advisory Board or its Educational Review Working Group. Both committees are staffed either by pulmonologists, primary care physicians or internal medicine doctors. Additionally, the site is monitored by a respiratory educator (who is also a COPD patient) whose role is to share experiences and appropriate resources and to resolve any inappropriate conversations or content. Although medical information is not dispensed on the site, the community moderator at times will solicit input from either the COPD Foundation's Chief Medical Director or Chief Scientific Officer to provide greater insight into conversations or discussions. Another tool for facilitation of appropriate monitoring of information includes the ability for community members to report a post that may be inappropriate, and the ability for community members to privately connect to other members of the community. Integrated analytics features provide community managers insight into user engagement and adoption, popular and trending content, influence scores, and user registration rates. In addition, the COPD Foundation staff, including 
respiratory therapists, public policy experts and researchers, provide non-medical, but valuable insight into many questions posed on the site. In a recent article by Stellefson et al, COPD360social is described as "uniquely blending transactional computermediated communication techniques with evidencebased processes for creating and delivering chronic disease self-management education."7

In addition to providing education and a source of camaraderie for patients and families, the COPD360social platform serves as a tool in the cultivation of COPD patients to support and participate in meaningful research. It also serves as a foundation for the collection of real-world data, helping us turn anecdotes into signals for research. The concept that online communities cannot only impact research, but can help drive patient-centric research is novel and is increasingly becoming an expectation from possible supporters. ${ }^{8}$ For example, in 2014, Genetech signed a 5-year data access deal with the online patient community "Patients Like $\mathrm{Me}$ " to address "persistent problems in its clinical trials." 9 While perhaps not the original intent of COPD360social or BronchandNTM360social, the concept that the community platform can provide a forum for research is exciting and emerging. As an example, COPD360social has been used to test an idea or theory with a simple post on the site. Should they choose to, patients can respond to a concept and the rapid response and ability to collect data quickly can save researchers hours of guesswork. In addition, COPD360social may be utilized more formally in research as well. The ability to conduct surveys, access private online focus groups and utilize a resource for dissemination of research via online social platforms is a growing interest for researchers. It is important to note that all research, should, of course, be conducted with the proper regulatory pieces in place.

We return to our original question, "Is there evidence to suggest that we should not just 'accept the existence of' online health care communitiesbut rather, embrace them for the education, peerto-peer support and other opportunities they offer?" It is the opinion of the authors that if information is provided by reputable sources and monitored to ensure quality, the answer is indisputable. Not only is access to online health information and communities inevitable, it is also invaluable and has only begun to educate, engage and empower patients---and---to revolutionize how we currently view health care.

\section{Acknowledgements}

The authors would like to acknowledge and thank William Clark, Delia Prieto and Gretchen McCreary for the exceptional job they do daily in making the COPD Foundation's online communities educational, supportive destinations for individuals suffering from COPD and bronchiectasis/NTM. The authors thank Byron Thomashow, MD and Barbara Yawn, MD for the continual guidance, perspective and leadership they provide not only to COPD360social, but to the COPD community in general. The authors also acknowledge and thank the late John W. Walsh for his never-ending passion and quest for new ideas and his foresight and trust of evolving technologies which allowed COPD360social to become a reality. 


\section{References}

1. Solberg LB. The benefits of online health communities. $A M A$ Journal of Ethics website. https://journalofethics.ama-assn.org/ article/benefits-online-health-communities/2014-04

Published April 2014. Accessed September 2018.

2. Fox S, Duggan M. Health online 2013. Pew Research Center internet and technology website. http://www.pewinternet. org/2013/01/15/health-online-2013/

Published April 2013. Accessed September 2018.

3. Mahler DA, Cerasoli F, Della L, Rudzinski M. Internet health behaviors of patients with chronic obstructive pulmonary disease and assessment of two disease websites. Chronic Obstr Pulm Dis. 2018;5(3):158-166.

doi: http://doi.org/10.15326/jcopdf.5.3.2017.0173

4. IntakeQ. Online patient communities: are they worth your time? IntakeQ website. https://blog.intakeq.com/online-patientcommunities-are-they-worth-your-time/

Published January 2017. Accessed September 2018.

5. Raine L, Fox S. The online health revolution. Pew Research Center internet and technology website. http://www.pewinternet. org/2000/11/26/the-online-health-care-revolution/

Published November 2000. Accessed September 2018.

6. Bruss L. Five concerns docs have about online patient communities. Ragan's Healthcare Communication News website. https://www.healthcarecommunication.com/Main/ Articles/5_concerns_docs_have_with_online_patient_ communiti_7857.aspx

Published October 2012. Accessed September 2018.

7. Stellefson M, Paige SR, Alber JM, Stewart M. COPD360social online community: a social media review. Health Promot Pract. 2018;19(4):489-491.

doi: https://doi.org/10.1177/1524839918779567

8. Bhomick A. Online health communities: a new frontier in health research. Medium website.https://medium.com/@abhowmick1/ online-health-communities-a-new-frontier-in-health-research71fb73edbea2

Published August 2016. Accessed September 2018.

9. Comstock J. How Genentech taps into online patient communities to improve clinical trials. MobiHealthNews website. https://www.mobihealthnews.com/36739/how-genente ch-taps-into-online-patient-communities-to-improve-clinicaltrials

Published September 2014. Accessed September 2018. 